\title{
Comparison of Mycobacteria Growth Indicator Tube with BACTEC 460 for Detection and Recovery of Mycobacteria from Clinical Specimens
}

\author{
F. ZUHRE BADAK,${ }^{1} \dagger$ DEANNA L. KISKA,${ }^{1} \ddagger$ SHARON SETTERQUIST,${ }^{1}$ CAMI HARTLEY,${ }^{1}$ \\ MICHAEL A. O'CONNELL, ${ }^{2}$ AND ROY L. HOPFER ${ }^{1,3,4 *}$ \\ Clinical Microbiology-Immunology Laboratories, University of North Carolina Hospitals, ${ }^{1}$ and Departments of \\ Microbiology-Immunology ${ }^{3}$ and Pathology, ${ }^{4}$ University of North Carolina School of Medicine, Chapel Hill, and Becton \\ Dickinson Research Center, Research Triangle Park, ${ }^{2}$ North Carolina
}

Received 16 January 1996/Returned for modification 13 May 1996/Accepted 26 June 1996

\begin{abstract}
We compared the Mycobacteria Growth Indicator Tube (MGIT) system with the BACTEC 460 (B460) and Lowenstein Jensen (LJ) systems for the recovery of mycobacteria (acid-fast bacteria [AFB]) from 1,441 clinical specimens. Excluding 13 isolates of Mycobacterium gordonae, 178 significant AFB isolates were recovered from 113 patients. Isolates (119) of the Mycobacterium avium complex (MAC) accounted for $67 \%$ of all isolates, while isolates (30) of the Mycobacterium tuberculosis complex (MTB) accounted for $17 \%$ of isolates. The MGIT system recovered $98(82 \%)$ MAC and $27(90 \%)$ MTB isolates, while the B460 system recovered $101(85 \%)$ MAC and $28(93 \%)$ MTB isolates and the LJ system recovered $91(76 \%)$ MAC and $25(83 \%)$ MTB isolates. Overall, the MGIT system recovered 152 isolates of AFB (85.4\% sensitivity), and the B460 and LJ systems recovered 151 (84.8\% sensitivity) and 137 (76.9\% sensitivity) AFB isolates, respectively. The recoveries of AFB with combinations of media were as follows: MGIT + LJ, 93.2\%; B460 + LJ, 92.1\%; and MGIT + B460, 96.6\%. Although the sensitivity of MGIT was equivalent to that of $B 460$, MGIT required a longer incubation (median, 11 days) than did B460 (median, 8 days) to become positive $(P<0.05)$.
\end{abstract}

During the past decade, there has been an increase in the incidence of tuberculosis in the United States. Coupled with the specter of multiple drug resistance in strains of the Mycobacterium tuberculosis complex (MTB), the need for more rapid diagnostic tests for early detection of MTB in clinical specimens has become even more apparent. Recent Centers for Disease Control and Prevention guidelines recommend laboratory identification of MTB from clinical specimens within 21 days (4). Although a variety of nucleic acid-based amplification methods have shown the potential for providing such information within 24 to $48 \mathrm{~h}$, many laboratories rely on culture methods for both primary identification and antimicrobial agent assessment. Therefore, an ideal system or medium for the culture of MTB and other acid-fast bacteria (AFB) should be sensitive and provide detection in the shortest possible incubation time. However, with increasing emphasis on reducing health care costs, the ideal culture system should also be inexpensive in terms of both the cost of materials and labor intensity.

Several commercial systems, as well as conventional media, are available for the culture of AFB. One commercial system, BACTEC 460 (B460), which detects the production of ${ }^{14} \mathrm{C}$ labeled $\mathrm{CO}_{2}$ as an early indicator of the growth of AFB has evolved to become the "gold standard" by which other culture systems are evaluated $(1-3,6,8,9,13)$. In addition to using at least one liquid medium, many laboratories inoculate a solid medium such as a Lowenstein Jensen (LJ) slant or Middlebrook agar. B460 has proven to be sensitive and to provide

\footnotetext{
* Corresponding author. Mailing address: UNC Hospitals, Clinical Microbiology, CB7600, Chapel Hill, NC 27514. Phone: (919) 966-7894. Fax: (919) 966-0486.

$\dagger$ Present address: Ege University, Izmir, Turkey.

$\ddagger$ Present address: State University of New York, Health Science Center, Syracuse, N.Y.
}

early detection of growth, and it is routinely used to obtain initial antimycobacterial agent sensitivities for MTB (5). The major drawbacks of the B460 system are well-known and include, (i) the potential for crosscontamination between culture bottles, (ii) the potential of aerosolization during the monitoring of culture bottles, (iii) the problems related to the use of radioactive materials, and (iv) labor requirements related to loading and unloading bottles and performing maintenance of the instrument. Preliminary studies evaluating the BBL Mycobacteria Growth Indicator Tube (MGIT) as a nonradiometric broth method for the growth and detection of mycobacterial isolates from clinical specimens and as a susceptibility testing method have been reported $(3,6,7,9)$. The MGIT system consists of Middlebrook $7 \mathrm{H} 9$ broth and a fluorescent compound embedded in silicone. Antibiotic and growth supplements are added to both MGIT and B460 prior to inoculation. Positive cultures are detected visually because of the metabolic depletion of oxygen, which otherwise quenches fluorescence. Instrumentation is not required, and multiple tubes can be inspected simultaneously. This study compares MGIT with B460 and LJ slants for the recovery of mycobacteria from clinical specimens.

(This study has previously been presented in part [8a].)

\section{MATERIALS AND METHODS}

All clinical specimens, except blood samples, submitted for AFB culture were included in this study. Standard $N$-acetyl-L-cysteine sodium hydroxide digestion, decontamination, and concentration methods (10) were used on nonsterile source specimens. All stool specimens and respiratory specimens from patients with cystic fibrosis were further decontaminated with oxalic acid (14). MGITs and $\mathrm{B} 460$ bottles (12B vials) received equal volumes $(0.5 \mathrm{ml})$ of specimen, while two LJ slants were each inoculated with $0.1 \mathrm{ml}$ of specimen. The majority of specimens were processed within $24 \mathrm{~h}$, and smears were prepared by fluorochrome staining (10). MGITs were read daily for 8 weeks. Positive MGITs were detected by bright orange fluorescence in the bottom of the tube and orange fluorescence on the air-broth meniscus. B460 readings were taken twice weekly for 2 weeks and once weekly for 2 additional weeks, and LJ slants were examined 
TABLE 1. Smear and culture results for 178 positive cultures

\begin{tabular}{|c|c|c|c|c|c|c|c|c|}
\hline \multirow{2}{*}{$\begin{array}{l}\text { Smear } \\
\text { result }\end{array}$} & \multirow{2}{*}{$\begin{array}{l}\text { No. of } \\
\text { smears }\end{array}$} & \multicolumn{3}{|c|}{ No. of positive cultures (\% recovery) } & \multicolumn{4}{|c|}{ No. of organisms isolated } \\
\hline & & MGIT & B460 & LJ & MTB & MAC & $\begin{array}{c}\text { Rapid } \\
\text { growers } a\end{array}$ & Other $^{b}$ \\
\hline Negative & 85 & $64(75.2)$ & $62(72.9)$ & $50(58.8)$ & 4 & 70 & 4 & 7 \\
\hline \multicolumn{9}{|l|}{ Positive } \\
\hline Total & 93 & 88 (94.6) & 89 (95.7) & 87 (93.5) & 26 & 49 & 15 & 3 \\
\hline $1+$ & 27 & 25 (95.6) & 25 (95.6) & $23(85.1)$ & 1 & 19 & 6 & 1 \\
\hline $2+$ & 34 & $33 \quad(97)$ & $32(91.4)$ & $32(94.1)$ & 10 & 20 & 4 & 0 \\
\hline $3+$ & 32 & $30(93.7)$ & $32(100)$ & $32(100)$ & 15 & 10 & 5 & 2 \\
\hline Total & 178 & $152(85.4)$ & $151(84.8)$ & $137(76.9)$ & 30 & 119 & 19 & 10 \\
\hline
\end{tabular}

${ }^{a}$ Includes 14 M. abscessus, 4 M. chelonae, and 1 M. fortuitum isolates.

${ }^{b}$ Includes six M. xenopi, two M. kansasii, one M. marinum, and one M. scrofulaceum isolates.

weekly for 8 weeks. MGITs and LJ slants from smear-positive specimens were held for up to 12 weeks. B460 readings were taken daily on specimens determined to be positive in MGITs. Isolates of AFB were identified by standard biochemical tests (10) and DNA-RNA hybridization (Gen-Probe).

The sensitivities of the three systems individually and in pairs are summarized as percentages of total positives detected by any method. The specificities of the individual systems are summarized as the number of false negatives split by isolate. The specificities for pairs are indicated via the number of positives for single systems. Separate summaries of contamination are provided. The sensitivities and specificities of these systems were compared by way of chi-square tests using just the total positives detected by all of the methods; the sample sizes were too small to break this comparison down by isolate.

The incubation times are summarized as box plots of the number of days to positive growth split by the three systems, with isolates grouped as total, Mycobacterium avium complex (MAC), MTB, rapid growers, and other. The solid box in each box plot indicates the middle half of the data, and the line inside the box represents the median. Extreme points are also highlighted. Box plots show the locations and spread of the data and indicate skewness. Given the skewed nature of the growth data, approximate confidence intervals for the median number of days to positive growth were constructed on the basis of the Wilcoxon statistic and used to compare these systems.

\section{RESULTS}

A total of 1,441 clinical specimens collected from 826 patients were included in this study. The majority of specimens $(1,134)$ were from respiratory sources, with sputum samples accounting for 649 specimens. Smear examinations were positive for AFB for 93 of the 178 culture-positive specimens (52\% sensitivity). The smear and culture results for all specimens with a positive smear or culture result are shown in Table 1. As shown, AFB grew from 93 of 105 smear-positive specimens. The 12 smear-positive culture-negative specimens included 6 specimens from patients with previously positive findings and 6 specimens from patients with no history or clinical indication of mycobacterial infection. In addition to the 93 smear-positive culture specimens, there were 85 smear-negative specimens that subsequently grew significant AFB. This results in a specificity of $99.5 \%$ and a sensitivity of $53.8 \%$ for smear examination.

The three culture systems were equivalent in the ability to culture organisms from smear-positive specimens (93 to $96 \%$ ), while the culture sensitivity from smear-negative specimens was $75.3 \%$ (64 of 85) for MGIT, 72.9\% (62 of 85) for B460, and $58.8 \%$ (50 of 85 ) for LJ. The overall sensitivities ranged from 76.9 (LJ) to 84.8 (B460) and $85.4 \%$ (MGIT). The MGIT and B460 sensitivities were not significantly different overall, and both showed significantly higher sensitivity than did LJ $(P<$ $0.05)$. The use of one LJ tube rather than two tubes would have reduced LJ sensitivity to approximately $61 \%$.

During this study, $86.7 \%$ (26 of 30) of specimens which subsequently grew MTB were smear positive, 15 of which were read as $3+$. The sensitivity of microscopic examination during this study exceeded similar results from longer studies at our institution, in which the smear-positive rate was $68 \%$ for specimens culture positive for MTB (unpublished data). Only $41.2 \%$ of specimens culture positive for MAC were smear positive, while 18 of 29 (62.1\%) specimens were smear positive when other AFB were isolated.

Of the 178 specimens that grew AFB, the MGIT, B460, and LJ results were culture negative on 26,27 , and 41 occasions, respectively (Table 2). Alternatively, AFB were grown from only one culture system on 14, 10, and 6 occasions from MGIT, B460, and LJ, respectively. As shown, MAC isolates accounted for the majority of culture discrepancies. Although the sample sizes for individual organism groups were too small for reasonable statistical testing, MGIT did appear to perform better for the culture of rapid growers. The majority (28 of 30) of AFB isolates that grew in only one of the three culture systems were from smear-negative specimens.

The time required for each culture system to detect the growth of the 178 culture-positive isolates is shown in a box plot (Fig. 1). The differences expressed by the growth of all AFB were a reflection of those found with MAC. The median time for the B460 system to detect MAC was lower than that of MGIT $(P<0.05)$. The median times for both B460 and MGIT systems to detect growth were markedly shorter than that of $\mathrm{LJ}(P<0.01)$. As shown, continued incubation of MGITs beyond 40 days resulted in the detection of only three additional isolates ( 3 of 152). The detection of all B460 isolates within 28 days reflects the 4-week incubation period of these bottles.

The levels of false-positive culture results due to the culture of fungi or bacteria other than AFB are shown in Table 3. LJ

TABLE 2. False-negative results and growth in only one culture system

\begin{tabular}{|c|c|c|c|c|c|c|c|}
\hline \multirow{2}{*}{ Organism } & \multirow{2}{*}{$\begin{array}{l}\text { No. of } \\
\text { positive } \\
\text { cultures }\end{array}$} & \multicolumn{3}{|c|}{$\begin{array}{l}\text { No. of negative } \\
\text { cultures }\end{array}$} & \multicolumn{3}{|c|}{$\begin{array}{l}\text { No. of cultures } \\
\text { positive only in: }\end{array}$} \\
\hline & & MGIT & B460 & LJ & MGIT & B460 & LJ \\
\hline MAC & 119 & 21 & 18 & 28 & 8 & 9 & 5 \\
\hline МТВ & 30 & 3 & 2 & 5 & 0 & 1 & 1 \\
\hline Rapid growers ${ }^{a}$ & 19 & 0 & 4 & 3 & 3 & 0 & 0 \\
\hline Other $^{b}$ & 10 & 2 & 3 & 5 & 3 & 0 & 0 \\
\hline Total & 178 & 26 & 27 & 41 & 14 & 10 & 6 \\
\hline
\end{tabular}

${ }^{a}$ See Table 1 , footnote $a$

${ }^{b}$ See Table 1 , footnote $b$ 


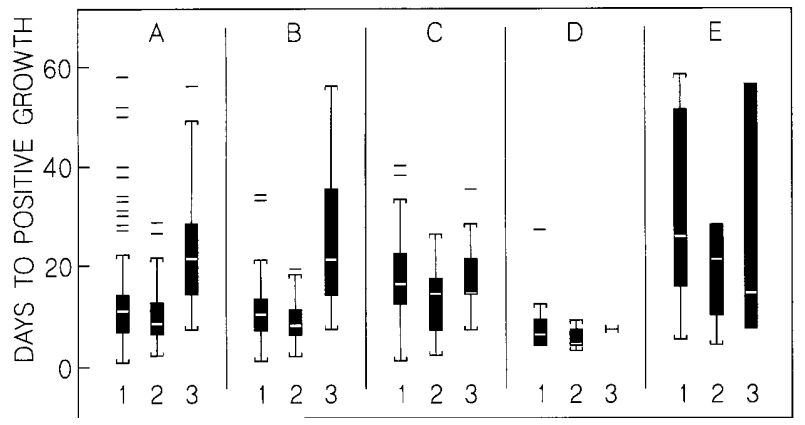

FIG. 1. Box plots for the time required to detect the growth of all AFB (A), MAC isolates (B), MTB isolates (C), rapid growers (D), and other AFB (E). Bars 1, 2, and 3 indicate the results for MGIT, B460, and LJ, respectively. Each solid box indicates the middle half of the data, and the line inside each box represents the median. Extreme points (lines) are shown. "Whiskers" are drawn to the nearest value not beyond 1.5 times the interquartile range from the quartiles.

slants had the highest rate of contamination (6.7\%), while MGIT and B460 had contamination rates of 5.6 and $3.9 \%$, respectively. MGITs contain the same antibiotics as do B460 bottles, but the concentrations range from 1.4 to 2.5 times higher than those in B460 vials. All MGIT and B460 bacterial and fungal contaminants were detected within the first 3 weeks of incubation. In addition to MGIT false-positive results due to the growth of contaminants, there were 50 MGITs $(3.5 \%$ of total) that became falsely positive (fluoresced) with no organisms seen on smear or subculture. These false-positive reactions occurred on average by day 13 .

\section{DISCUSSION}

The B460 system has become the standard with which other mycobacterial culture media systems are compared. In a recent College of American Pathologists Q-Probe survey (5), 56.9\% of the 534 institutions participating in the survey reported their use of B460. In addition, most (95\%) of these same laboratories reported the use of conventional solid media for primary isolation of mycobacteria. Liquid-based media such as B460 and Septi-Chek (BBL) have generally been found to be more sensitive and require fewer days to become positive than do conventional solid media $(2,11)$. This study compared the sensitivity and time to detection of MGIT with those of both B460 and LJ slants, a solid conventional medium. The MGIT system, similar to B460, detects growth by metabolic changes that occur in the medium. In both cases, AFB should be detected in less time than that needed to detect visible growth (turbidity) in liquid media or the appearance of colonies on solid media.

The study design used in this investigation was somewhat biased in favor of the MGIT system in that MGITs were read daily for 8 weeks while B460 bottles and LJ slants were read less frequently. Additionally, B460 bottles were incubated for only 4 weeks. However, as mentioned earlier, B460 bottles

TABLE 3. False-positive culture results

\begin{tabular}{lcccc}
\hline \multirow{2}{*}{ System } & \multicolumn{4}{c}{ No. of false-positive cultures } \\
\cline { 2 - 5 } & Bacteria & Fungi & No Growth & Total \\
\hline MGIT & 72 & 9 & 50 & 131 \\
B460 & 48 & 8 & 0 & 56 \\
LJ & 85 & 12 & 0 & 97 \\
\hline
\end{tabular}

were monitored daily after a positive result in the MGIT system. The study design could also account for the decreased sensitivity of the LJ slants since each slant received approximately $0.1 \mathrm{ml}$ of specimen versus the $0.5-\mathrm{ml}$ aliquots inoculated into MGIT and B460. Under the conditions of this study, the MGIT system compared favorably with B460 in terms of the sensitivity of mycobacterial culture ( 85.4 versus $84.8 \%$ ).

Not surprisingly, the culture failures of all three systems were primarily from smear-negative specimens (Table 1 ). The use of LJ slants increased the yield to 93.2 and $92.1 \%$ for MGIT and B460 broth systems, respectively. In other words, using either MGIT or B460 without a solid medium would have decreased the sensitivity of culture by 7 to $8 \%$, similar to the 4 to $6 \%$ reported by others (12). The equivalency in the total misses by MGIT and B460 (26 versus 27$)$, as well as the cultures positive only by MGIT or B460 (14 versus 10), suggests that small numbers of organisms present in these specimens were responsible for culture-negative differences rather than system failures of MGIT or B460 per se.

In addition to culture sensitivity, an equally important criterion of system performance is the incubation time required prior to the detection of growth. Although the median time to detect a positive culture was slightly less $(P<0.05)$ for B460 than for MGIT (8 versus 11 days), both were considerably faster $(P<0.01)$ than LJ slants $(21$ days $)$. MAC accounted for $66.8 \%$ of isolates, and the distribution of times for the detection of MAC was nearly identical to that required for all AFB (Fig. 1). Interestingly, LJ slants were nearly equivalent to MGIT and B460 in the length of incubation needed to detect MTB isolates (16 versus 14 days); however, LJ culture sensitivity for MTB was less $(83.3 \%)$ than that of either MGIT $(90 \%)$ or B460 (93.3\%). Although we continued MGIT incubation for 8 weeks in this study, only three AFB (two M. xenopi and one M. kansasii) isolates were detected after 6 weeks of incubation. B460 failed to grow any M. gordonae isolates, while MGIT grew 7 and LJ slants grew 13 isolates. Since all these isolates were considered contaminants and the patients were not treated with antimycobacterial agents, these isolates were not included in this evaluation. One could argue, however, that detecting these isolates increases the laboratory workload and expenses, with no improvement in patient care. In order to reduce the labor associated with reading $\mathrm{LJ}$ slants, one could also argue that LJ slants need not be read until after 3 weeks of incubation. During this study, a delay in reading LJ slants until the third week would not have significantly reduced the time to detection for either the MGIT-LJ or B460-LJ combination compared with the results obtained with MGIT and B460 alone.

In terms of excess labor required to deal with false-positive results (contaminants and/or fluorescence), B460 was superior to MGIT. B460 grew 56 non-AFB organisms, while MGIT grew 81 . This is somewhat surprising since MGIT contains the same antibiotics as do B460 bottles at 1.4- to 2.5-times-higher concentrations. Although B460 bottles were incubated for 4 weeks compared with the 8-week MGIT incubation, all of the MGIT contaminants were detected during the first 21 days of incubation. Fluorescent MGITs with negative smears and cultures occurred for 50 additional cultures. Although this number appears to be small, it increased the total number of smears and subcultures of MGITs from 233 (152 true positives plus 81 contaminants) to 283 , a $21.4 \%$ increase in labor. In total, the additional labor resulting from contamination and false fluorescence with MGIT was $86 \%$ above the labor required for true positives, while contamination in B460 increased labor by $37 \%$.

Finally, the MGIT system is more expensive in terms of 
material costs per culture than the B460 system ( $\$ 6.88$ versus $\$ 2.12)$. However, B460 requires expenses associated with the upkeep and maintenance of the monitoring device, disposal of radioactive wastes, and labor associated with regular loading, preparation of bottles for monitoring, and unloading of bottles. The labor associated with MGIT manipulation and the expenses for upkeep and maintenance of a UV fluorescent lamp are considerably less than that for B460.

In conclusion, the sensitivity and time to detection of MGIT were comparable to those of B460, although MGIT did require a slightly longer time to detection. The additional MGIT incubation times reflected in the median times to a positive result were most notable in the detection of AFB other than MTB, MAC, or rapid growers. Since there were only 10 isolates in this group, these differences are of questionable significance. Obviously, from an infection control standpoint, the more critical information is the speed of MTB detection. In this study, the median times to detect MTB were only 16, 14, and 14 days for MGIT, B460, and LJ slants, respectively. Others $(2,11)$ have shown that B460 requires much less time to detect MTB than do LJ slants (15 versus 17 to 25 days). Our LJ data probably reflect the fact that 25 of 30 cultures that grew MTB were read as $2+$ or $3+$ on smear examination, while at the same time, LJ slants failed to grow 5 of these 30 isolates (4 were smear negative, and 1 read as $1+$ ). Although MGIT performed well in this study, laboratories necessarily have to select AFB culture systems most amenable to their specific laboratory needs on the basis of additional criteria, including overall expenses, staffing, and work flow considerations.

\section{ACKNOWLEDGMENT}

This work was supported in part by Becton Dickinson Research Center, Research Triangle Park, N.C.

\section{REFERENCES}

1. Abe, C., S. Hosojima, Y. Fukasawa, Y. Kazumi, M. Takahashi, K. Hirano, and T. Mori. 1992. Comparison of MB-Check, BACTEC, and egg-based media for recovery of mycobacteria. J. Clin. Microbiol. 30:878-881.

2. Anargyros, P., D. S. J. Astill, and I. S. L. Lim. 1990. Comparison of improved BACTEC and Lowenstein-Jensen media for culture of mycobacteria from clinical specimens. J. Clin. Microbiol. 28:1288-1291.

3. Bowles, E. J., T. R. Wisniewski, J. M. Makowski, K. K. Frantz, and J. A. Kiehlbauch. 1995. A clinical comparison of the BBL MGIT mycobacteria growth indicator tube with Bactec, Septichek, and conventional media, abstr. U-142, p. 142. In Abstracts of the 95th General Meeting of the American
Society for Microbiology 1995. American Society for Microbiology, Washington, D.C.

4. Centers for Disease Control and Prevention. 1992. National action plan to combat multidrug-resistant tuberculosis. Meeting the challenge of multidrug-resistant tuberculosis: summary of a conference. Morbid. Mortal. Weekly Rep. 41:55-56.

5. College of American Pathologists. 1994. Q-Probes. Laboratory diagnosis of tuberculosis. Data analysis and critique. College of American Pathologists, Chicago.

6. Evans, K., A. Nakasone, P. Sutherland, and E. Peterson. 1995. Evaluation of the mycobacterial growth tube (MGIT) for the recovery of Mycobacterium spp., abstr. C-139, p. 25. In Abstracts of the 95th General Meeting of the American Society for Microbiology 1995. American Society for Microbiology, Washington, D.C.

7. Hagemann, P. A., S. E. Kodsi, J. D. Douglass, and D. T. Stitt. 1995. Preliminary methods for streptomycin and ethambutol susceptibility testing of $M$. tuberculosis using the BBL MGIT mycobacteria growth indicator tube, abstr. U-65, p. 128. In Abstracts of the 95th General Meeting of the American Society for Microbiology 1995. American Society for Microbiology, Washington, D.C.

8. Isenberg, H. D., R. F. D'Amato, L. Heifets, P. R. Murray, M. Scardamaglia, M. C. Jacobs, P. Alperstein, and A. Niles. 1991. Collaborative feasibility study of a biphasic system (Roche Septi-Chek AFB) for rapid detection and isolation of mycobacteria. J. Clin. Microbiol. 29:1719-1722.

8a.Kiska, D. L., F. Z. Badak, S. Setterquist, C. Hartley, M. A. O'Connell, and R. L. Hopfer. 1996. Comparison of Mycobacterial Growth Indicator Tube (MGIT) with BacTec 460 for recovery of mycobacteria, abstr. C-13, p. 3. In Abstracts of the 96th General Meeting of the American Society for Microbiology 1996. American Society for Microbiology, Washington, D.C.

9. Neumann, M. A., J. Rogers, R. Williams, and S. Maxwell. 1995. Comparison of the new mycobacterial growth indicator tube (MGIT) with the Bactec 460 TB system for the rapid detection of mycobacteria from clinical specimens, abstr. C-138, p. 24. In Abstracts of the 95th General Meeting of the American Society for Microbiology 1995. American Society for Microbiology, Washington, D.C.

10. Nolte, F. S., and B. Metchock. 1995. Mycobacterium, p. 400-437. In P. R. Murray, E. J. Baron, M. A. Pfaller, F. C. Tenover, and R. H. Yolken (ed.), Manual of clinical microbiology, 6th ed. American Society for Microbiology, Washington, D.C.

11. Sewell, D. L., A. L. Rashad, W. J. Rourke, Jr., S. L. Poor, J. A. McCarthy, and M. A. Pfaller. 1993. Comparison of the Septi-Chek AFB and BACTEC systems and conventional culture for recovery of mycobacteria. J. Clin. Microbiol. 31:2689-2691.

12. Stager, C. E., J. P. Libonati, S. H. Siddiqi, J. R. Davis, N. M. Hooper, J. F. Baker, and M. E. Carter. 1991. Role of solid media when used in conjunction with the BACTEC system for mycobacterial isolation and identification. J. Clin. Microbiol. 29:154-157.

13. Whittier, P. S., K. Westfall, S. Setterquist, and R. L. Hopfer. 1992. Evaluation of the Septi-Chek AFB system in the recovery of mycobacteria. Eur. J. Clin. Microbiol. Infect. Dis. 11:915-918.

14. Whittier, S., R. L. Hopfer, M. R. Knowles, and P. H. Gilligan. 1993. Improved recovery of mycobacteria from respiratory secretions of patients with cystic fibrosis. J. Clin. Microbiol. 31:861-864. 